\title{
Residual Stresses Induced in T Butt Welds from Submerged Arc Welding of High Strength Thick Section Steel Members
}

\author{
G.W. Sloan ${ }^{1, a, ~}{ }^{\star}$, S. Pearce ${ }^{1, b}$, V.M. Linton ${ }^{2, c}$, X. Ficquet $^{3, d}$, E. Kingston ${ }^{3,}$, \\ I. Brown ${ }^{1, f}$ \\ ${ }^{1}$ School of Mechanical Engineering, University of Adelaide, North Terrace, Adelaide, Australia \\ ${ }^{2}$ Executive Dean Faculty of Engineering and Sciences, University of Wollongong, New South \\ Wales, Australia \\ ${ }^{3}$ Veqter Ltd, Unicorn Business Park, Whitby Road, Bristol, BS4-4EX, UK \\ aglen.sloan@bigpond.com.au, bsusan.pearce@adelaide.edu.au, cvalerie.linton@uow.edu.au, \\ dxavier.ficquet@veqter.co.uk, eed.kingston@veqter.co.uk, fian.bee13@gmail.com
}

Keywords: Residual Stress, High Strength Steel, Quenched and Tempered, Neutron Diffraction, Deep Hole Drilling, T Butt Welds

\begin{abstract}
The overall residual stress profiles of a weld fabricated structures such as a submarine pressure hull fabricated using high strength welds joining quench and tempered steels is complex as they are the net profiles from the build-up or relaxation of the residual stresses induced into the welds and structural members from each step in the fabrication process. The University of Adelaide with its research partners has attempted to increase the knowledge of residual stresses in this type of structure by undertaking a comprehensive residual stress measurement program using test pieces representing each stage of the fabrication of a submarine pressure hull including full scale test pieces. The primary methods of measuring subsurface residual stress profiles was the neutron diffraction method with the Deep Hole Drilling technique (DHD) to validate key results. The $\mathrm{X}$ ray diffraction method and Incremental Centre Hole Drilling methods were used for determining surface residual stresses. This paper presents an overview of the results for residual stress profiles resulting from a full penetration double sided $\mathrm{T}$ Butt weld joining a $\mathrm{T}$ Frame to a thick flat plate by Submerged Arc Welding. The test piece used a specific pass placement strategy that placed the last pass each side on the base plate. The results presented show that the actual profile is considerably different than those for established fracture mechanics residual stress profiles in that these are over conservative. The discussion will include comparison of results to other measurement results undertaken on similar test pieces but undertaken with an alternate pass placement strategy.
\end{abstract}

\section{Introduction}

Ring stiffened cylindrical structures for the offshore industry and naval platforms are usually fabricated from high strength steels with butt welds and $\mathrm{T}$ butt welds. The fabrication method involves manufacturing plate cans from thick plates with the longitudinal direction of initial rolling of the plate coinciding with that for shape rolling into a curve. Depending on the diameter 3 or more plates will be needed to be welded together to form a cylindrical can. The ring frames are also fabricated from high strength steel and are welded into the can via double sided $\mathrm{T}$ butt welds undertaken in the $1 \mathrm{G}$ down hand orientation. The same also applies for rigid bulkheads in the structures to resist buckling of the structures when subjected to sea pressure. These types of structures operate in environments where Stress Corrosion Cracking (SCC) is a frequent problem for crack initiation in service as is Hydrogen Assisted Cold Cracking (HACC) during manufacture. In the case of naval platforms they are also subjected to a high stress low cycle 
fatigue regime throughout their service life. Therefore an understanding of residual stress profiles is essential for determining service life and structural integrity safety regimes for the welds and the structural components. While standards such as BS 7910 [1] present residual stress profiles for $\mathrm{T}$ butt welds they are limited to the weld toe region only and they assume that the last weld pass is on the base plate with the residual stress level being at tensile yield. No profiles are given for the region under the weld or in the base plates away from the welds and how much the existing profiles in the components are altered by adjacent welding. Hence there is a huge gap in knowledge of residual stress profiles throughout a fabricated ring stiffened cylindrical structure. In 2004 the University of Adelaide and its research partners, ISIS Rutherford Appleton Laboratories, Australian Nuclear Science and Testing Organization, ASC Pty Ltd, and the Commonwealth of Australia, implemented a program to address these gaps [2], [3]. The program was structured to look at the progressive build up or superimposition of residual stress profiles throughout the fabrication process for these types of structures and addressed both current and potential future weld pass placement strategies for the $\mathrm{T}$ butt welds. The areas of primary interest were the base plate both beneath the weld and away from the weld region and also the weld. The material used throughout the entire program was BIS 812 EMA quenched and tempered plate with a minimum yield strength of $690 \mathrm{MPa}$ [2] and a Poisson's ratio of 0.3. This yield value was used to normalise all the results. The chemical composition is given in ref [2]. The Young's modulus for the material varies between 208 to $213 \mathrm{GPa}$. A value of $210 \mathrm{GPa}$ was used for the calculations of strain and stress. The test piece the subject of this paper was that for a weld of a heavy section frame to a flat plate with the welding being undertaken by the Submerged Arc Welding process (SAW).

\section{Experimental Methodology}

The flat $\mathrm{T}$ Butt test plate was designed to be representative of the welds joining stiffening frames to submarine flat plate rigid bulkheads and also of the pressure hull ring frames to curved shell plate welds but eliminating in this later case the variable of the pre-existing residual stresses from the curved shell plate prior to welding of the frame to shell which is in line with the logic presented in the previous section [2], [3]. These were normally undertaken using the SAW welding process in an automatic welding set up welding side 1 completely, back-gouging the root and then welding side 2 fully [2] to achieve a full penetration $\mathrm{T}$ butt weld. All welding was undertaken in the $1 \mathrm{G}$ orientation. The weld cap is a tapered weld, approximately $5^{0}$ to the base plate with the last pass on the base plate. This is a narrower taper than used in BS7910 [1]. The sample was 1 metre long. The brackets indicated in Fig 1 were welded to the base plate and the T Frame before the test weld of the T Frame to the base plate was undertaken to prevent distortion / rotation of the base plate relative to the $\mathrm{T}$ Frame and therefore eliminate a variable. All residual stress measurements were undertaken as close as possible to the mid length of the test piece. The welding of the web to base plate was undertaken using a maximum heat input of $1.9 \mathrm{~kJ} / \mathrm{mm}$ [2], [3] using Mil 120S-1 wire with OP121 TT flux. As SAW is an automatic process the heat input was constant thereby eliminating a variable. Reducing variables is a key approach factor to understand results.

The primary method selected for determining residual stresses in the subsurface regions of the fabricated test piece was the Neutron Diffraction (ND) method [3]. This was chosen as it is totally non-destructive and therefore allowed the subsequent options to use other destructive methods to verify/ validate the results or could be repeated if necessary. Five scan lines were undertaken as detailed in Fig 2 as detailed in [2]. The scan lines concentrated on obtaining strain and stress data across the plate and excluded the zone immediately under the weld due to the large beam path lengths for the longitudinal strain direction, which increase the risk of no effective measurements due to beam scatter, even with the sample tilted to $30^{\circ}$ which was done. 
The gauge volumes for the neutron diffraction were $4 \times 4 \times 20 \mathrm{~mm}$ for the transverse and normal directions and $4 \times 4 \times 4 \mathrm{~mm}$ for the longitudinal direction. The test piece was laser scanned and the resultant $3 \mathrm{D}$ model used to generate the sample plan in the computer program SScanSS developed by the Open University [4]. This sample plan was then used to drive the scanning table at Engine $\mathrm{X}$ at the Rutherford Appleton Laboratories UK. It has been optimized to reflect available beam time as opposed to full allocation.

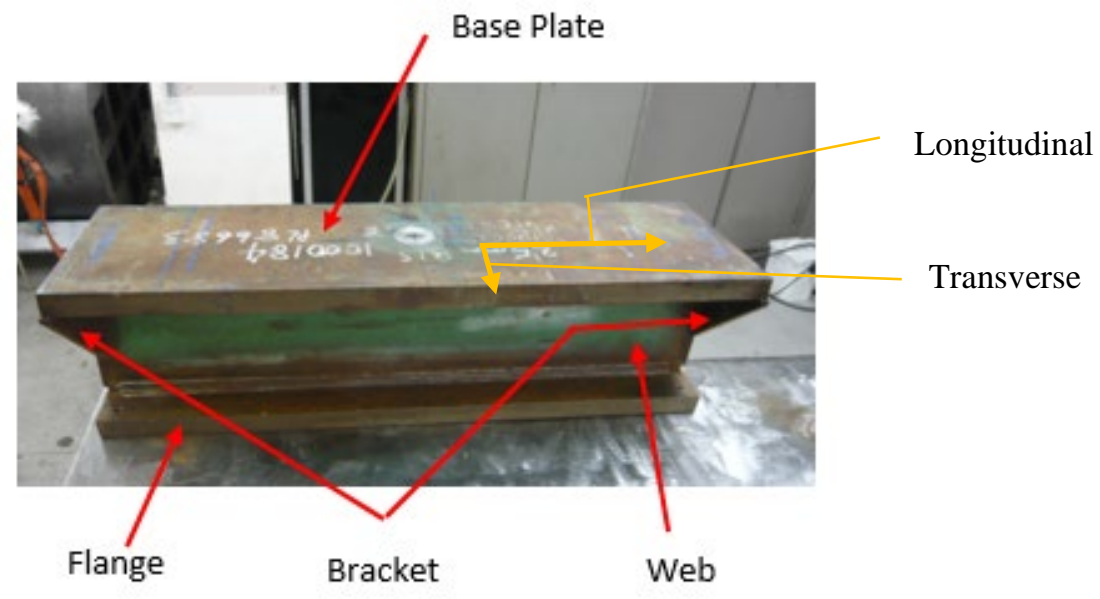

Fig. 1 Showing the design of the Flat $T$ butt test plate and the principal elements and residual stress directions. The longitudinal rolling directions for the plate components is also that for welding.

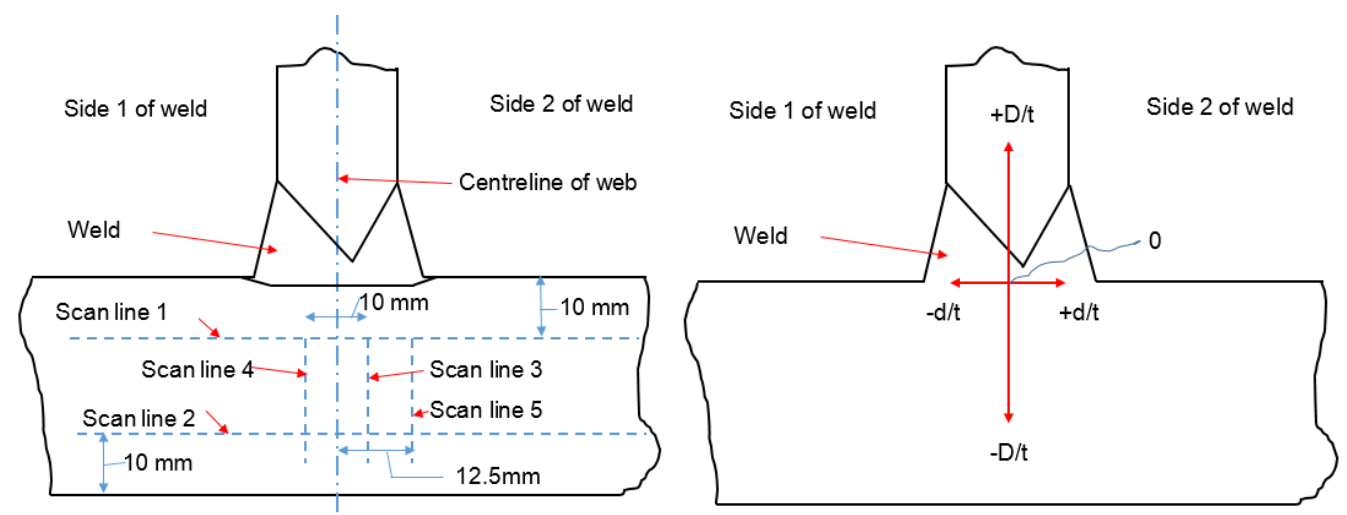

Fig. 2 Showing details of the scanlines for the neutron diffraction testing on the left and the normalized datum's used for result locations on the right. $D$ is the normalized depth through thickness i.e. Depth /base plate thickness $t$ and $d$ is the normalized distance from centerline of web $d /$ base plate thickness.

In order to validate the neutron diffraction work one scan line was tested by the iDHD/DHD technique. The iDHD/DHD technique is described in [5]. The location chosen was in line with Scan Line 5 of Pearce [2] as this is closest to the weld toe side 2 and therefore could be reviewed against BS 7910 profiles and other publications. As noted in Sloan [3] the gauge volumes for the ND and DHD techniques are slightly different but in this case the DHD measurements were directly centered on Scan line 5 and not offset as occurred in [3] therefore permitting easier comparison of results noting difference in gauge volumes. 


\section{Results}

The results presented in this paper will use the datum configuration as presented in Fig 2. extended up into the weld above the top of the base plate and is in line with those used in [3]. The results of ND scans for scan lines 1 and 2 Pearce [2] are shown superimposed in Fig 3. As can be seen in Fig 3 Scan line 1 closest to the web side was undertaken fully across the area of interest of the base plate whereas for Scan line 2 the measurements started at the through thickness scan line 3 and extended out onto side 2 of the weld. The longitudinal residual stresses are still tensile at $10 \mathrm{~mm}$ depth but revert to compressive at $25 \mathrm{~mm}$ depth. The opposite has been found for the transverse stress profiles. The error bars for the longitudinal stress scan line 1 under the weld reflect the issue of excessive beam path length.

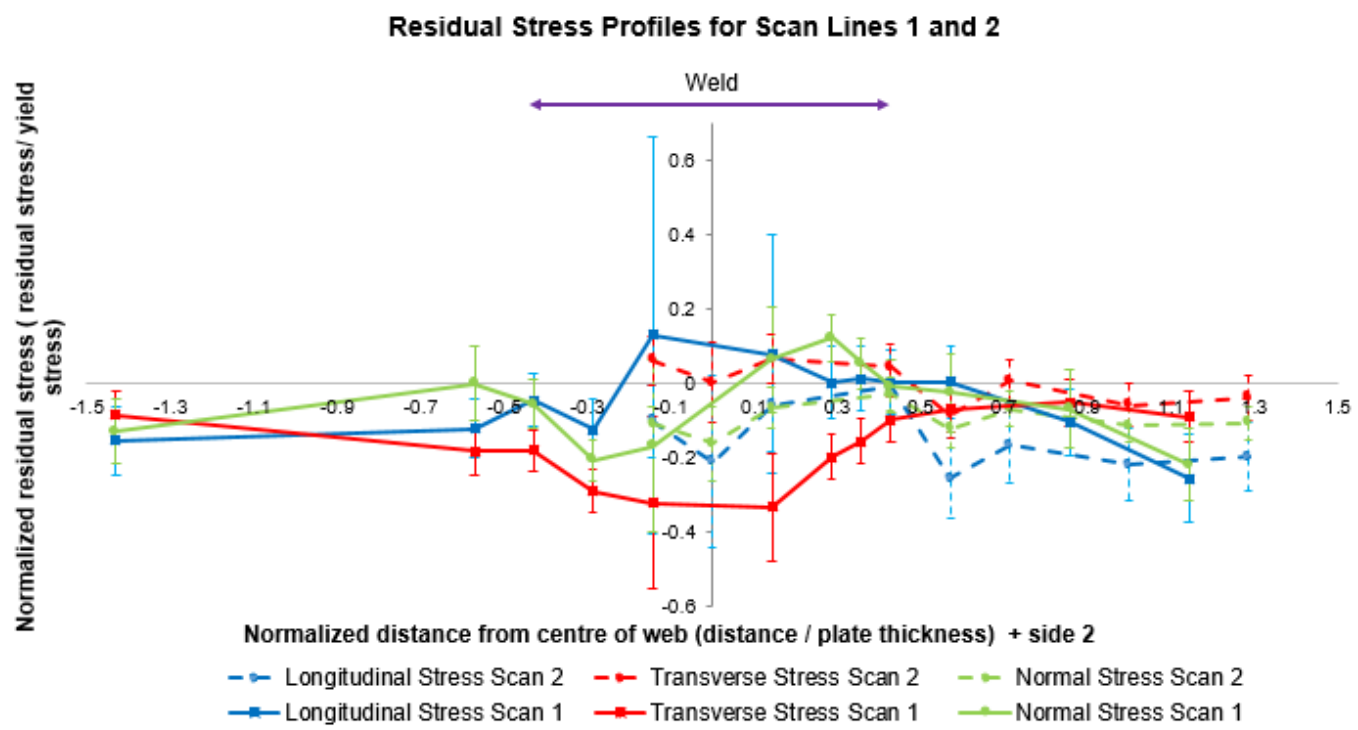

Fig. 3 Shows the plots of residual stress across the base plate for scan lines 1 and 2, see Fig 2, and Fig 1 for the principal directions in a normalized stress and distance d format. The large error bars for longitudinal stress under the weld reflect the excessive beam path lengths.
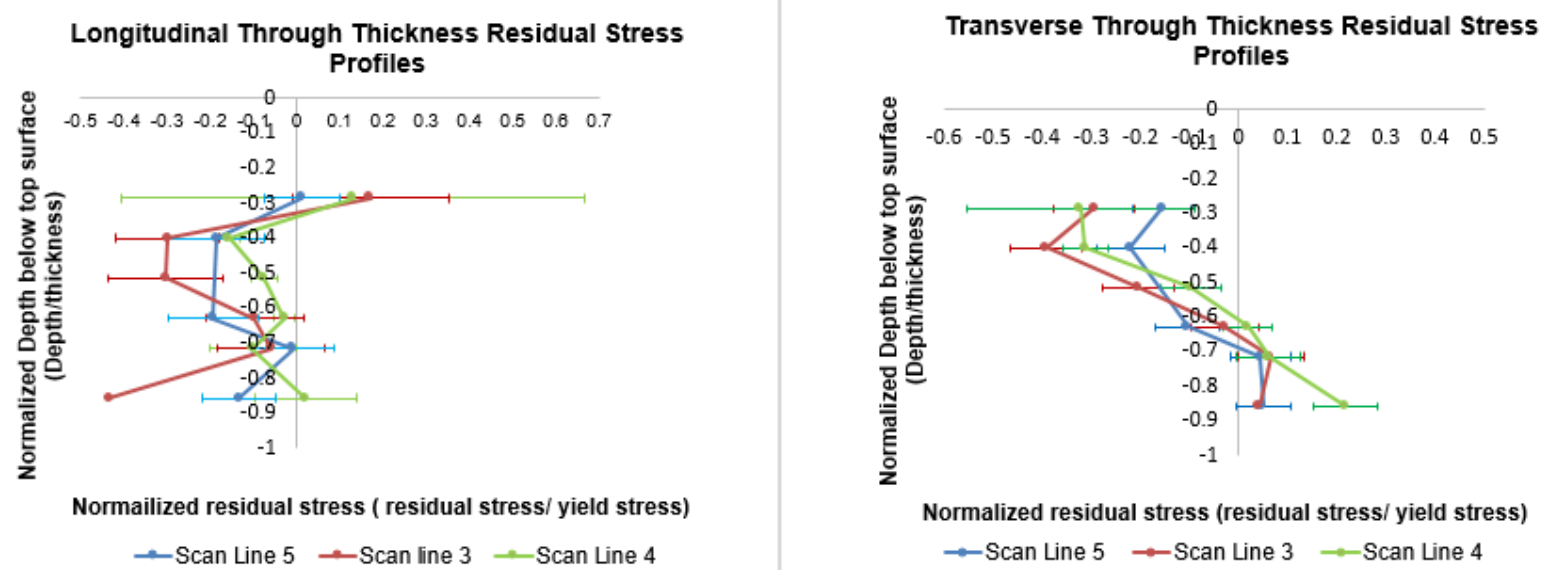

Fig. 4 Details the results for the longitudinal and transverse residual stress profiles for the scan lines identified in Fig 2 in a normalized stress and Depth D format.

The residual stress profiles for the normal direction are not shown in Fig 4 as they cannot be compared with results from the DHD/iDHD measurements undertaken on the test piece with the assistance of Veqter Ltd as it is effectively a 2 dimensional method. They can however be found in Pearce [2]. The results of the DHD/iDHD measurements are shown in Fig 5 below. You will observe that the results for transverse residual stress stop at the intersection with the web 
interface. Above this point the stresses determined by the DHD / iDHD technique become the longitudinal and the normal direction relative to the welding as described in Sloan [3]. The iDHD results closely match the DHD results and so there has not been significant impact of plasticity at or near the weld. The results are totally different from the profiles which are generated from [1], i.e. no yield level stresses at the weld toe.
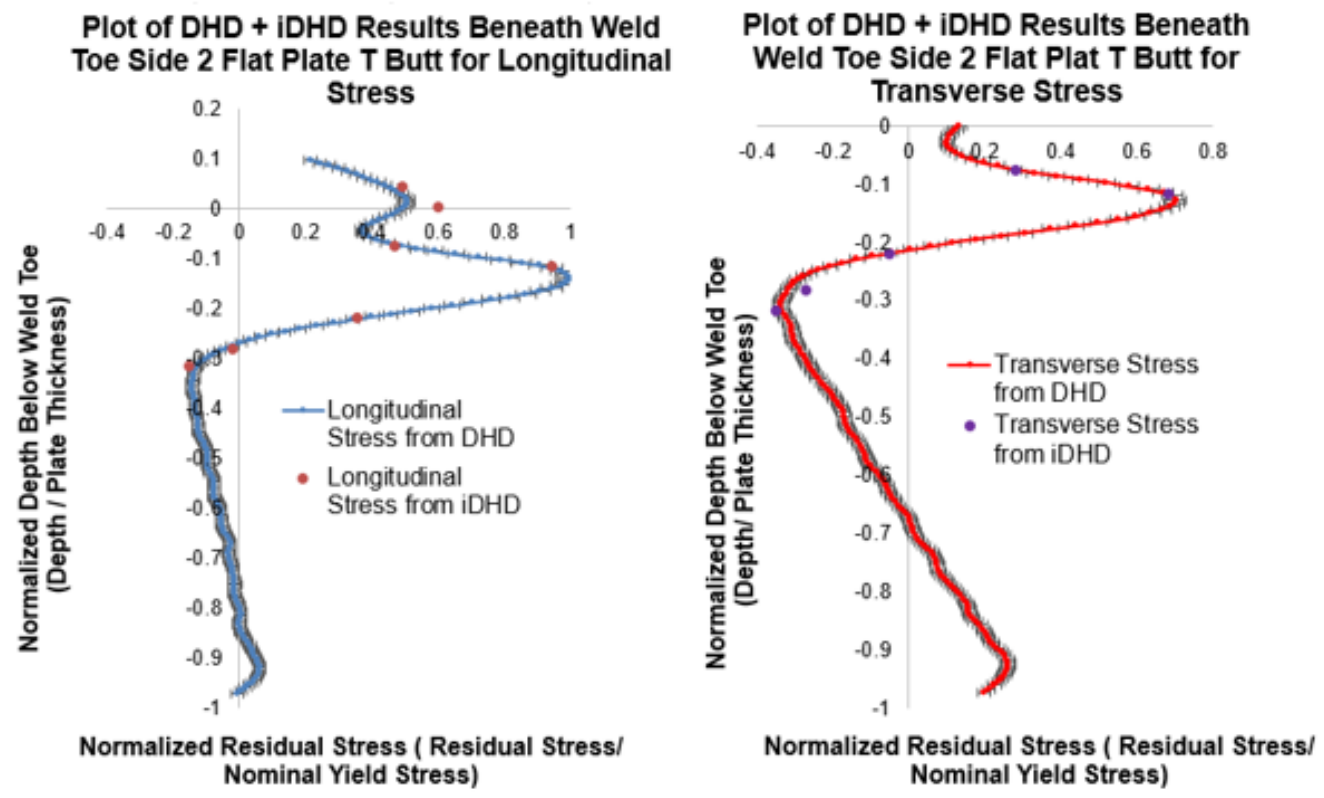

Fig. 5 Detailing the longitudinal and transverse residual stress profiles for the DHD and iDHD measurements of residual stress. The errors by this method are small. The normal stress direction in the base plate is not possible for this method as it iseffectively a $2 D$ method. The results are significantly different from the profiles generated from [1].

\section{Discussion}

The results presented above clearly highlight that the residual stress profiles detailed in [1] are conservative. The residual stress levels immediately under the weld toe are not tensile yield as per [1] and they change to compressive levels very quickly i.e. between 0.2 and $0.3 \mathrm{D} / \mathrm{t}$ which is also different than the predictive profiles of [1]. This was also the case for the results in [3] with a different weld pass placement strategy i.e. last pass on the web member.

In Fig 6 the results for the ND measurements [2] are superimposed on the DHD / iDHD results figure 5. These show very close correlation between results from the two different methods noting the slightly different gauge volumes, however there is that zone beneath the main portion of the T butt weld where there is no ND data which needs to be addressed by other alternative methods such as the Contouring method or Ultrasonic method.

\section{Conclusions}

The information presented above shows that the residual stress profiles detailed in [1] are very conservative and may lead to unnecessary weld repairs being implemented which may themselves effect integrity but will certainly effect cost, schedule and life of structure. Extensive further work needs to be undertaken in this field to highlight the extent of conservatism and provide a structured way forward particularly the zone immediately under the $\mathrm{T}$ butt weld. Critical methods to explore are those that can be undertaken on full scale structures, either in service or large scale test pieces, which do not involve cutting and therefore relaxing of residual stresses, and therefore the research trend should be towards methods such as ultrasonics provided that they are validated against known results. 

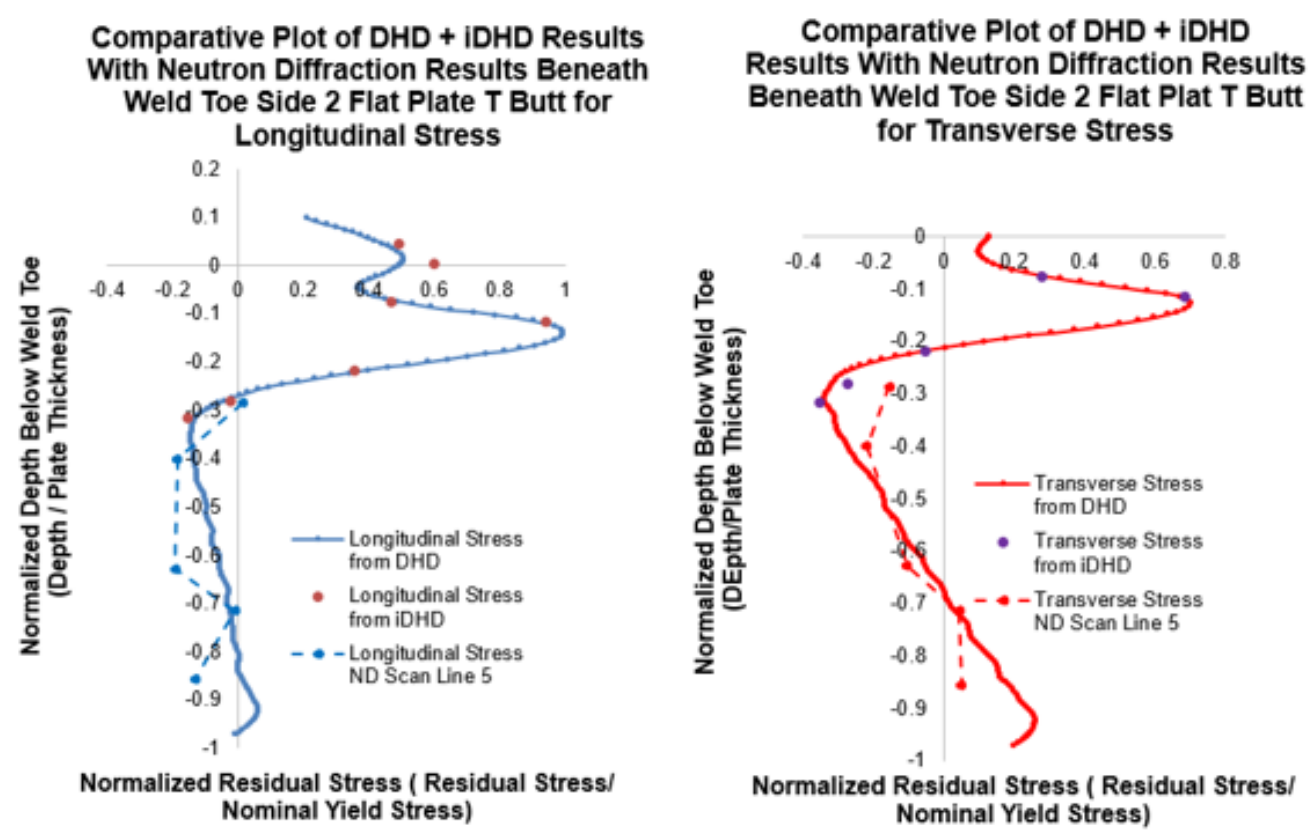

Fig. 6 Shows an overlay of the ND results as shown in Fig 4. for the longitudinal and transverse directions with those achieved via the DHD/ iDHD method for Scan Line 5.

\section{Acknowledgments}

The author wishes to acknowledge the support from the University of Adelaide, ASC Pty Ltd, and the Commonwealth of Australia, and Veqter Ltd. Most importantly the author wishes to acknowledge his parents whose estate funded the DHD work.

\section{References}

[1] BS 7910 "Guidance on methods of assessing the acceptability of flaws in metallic structures”, 1999 with amendments 2000, 2009

[2] S. Pearce "Breakdown of residual stresses in highly restrained thick section steel welds", Doctoral thesis, University of Adelaide 2009.

[3] G Sloan 'The influence of welding variables on the residual stress profiles around $\mathrm{T}$ butt and MMAW butt welds in thick section high strength steel”, Master's thesis, University of Adelaide 2015.

[4] J. A. James and L. Edwards "Application of robotic kinematics methods to the simulation and control of neutron beam line positioning systems”, Nuclear Instruments and Methods in Physics Research A, 2007. https://doi.org/10.1016/j.nima.2006.11.033

[5] Information on http://www.vqter.co.uk 